Research Article

\title{
Comparative Analysis of Virulence Factors of Homozygous and Heterozygous Strains of Candida albicans Vaginal Isolates
}

\author{
Hasti Nouraei, ${ }^{1}$ Sahar Sheykhi, ${ }^{1}$ Zahra ZareShahrabadi, ${ }^{1}$ Hossein Khodadadi, \\ Kamiar Zomorodian, ${ }^{1,2}$ and Keyvan Pakshir (D), \\ ${ }^{1}$ Department of Parasitology and Mycology, School of Medicine, Shiraz University of Medical Sciences, 7134845794, Shiraz, Iran \\ ${ }^{2}$ Basic Sciences in Infectious Diseases Research Center, School of Medicine, Shiraz University of Medical Sciences, 7134845794, \\ Shiraz, Iran
}

Correspondence should be addressed to Keyvan Pakshir; pakshirk@gmail.com

Received 26 April 2020; Accepted 9 June 2020; Published 27 June 2020

Academic Editor: Giuseppe Comi

Copyright $(92020$ Hasti Nouraei et al. This is an open access article distributed under the Creative Commons Attribution License, which permits unrestricted use, distribution, and reproduction in any medium, provided the original work is properly cited.

\begin{abstract}
Although the epidemiology of pathogenic Candida species is changing due to invasive diseases, Candida albicans has become the common cause of human infections worldwide. Candida albicans is a diploid yeast with a mostly clonal mode of reproduction and without known complete sexual cycle. This species has two heterozygous and homozygous strains at hyphal wall protein 1 gene locus ( $h w p 1)$. Little is known about virulence factors of these strains. The aim of this study was to evaluate the exoenzyme activity of heterozygous and homozygous C. albicans strains. A total of 60 stock Candida albicans species isolates, which consisted of 30 homozygous and 30 heterozygous strains, were used for exoenzyme activities. We used egg yolk agar, Sabouraud blood agar, and bovine serum albumin agar for evaluation of phospholipase, hemolysin, and proteinase activity, respectively. Homozygous strains of Candida albicans had more phospholipase and proteinase activity than heterozygous strains. However, there were no significant statistical differences between the two strains in the severity of exoenzymes production. Beta hemolysin activity was seen in $100 \%$ and $96.7 \%$ of the homozygous and heterozygous strains, respectively. The results of this study indicated that both of the strains exhibited exoenzyme activities in different ranges. There were no significant statistical differences in virulence factors between the homozygous and heterozygous strains.
\end{abstract}

\section{Introduction}

Candida albicans is a commensal diploid organism and inhabits a variety of niches in human populations. It becomes an opportunistic pathogen in immunocompromised patients and can cause a wide variety of infections, ranging from superficial to disseminated infections. Candida albicans is the common fungal pathogen and has advanced a range of assumed virulence factors that allow successful colonization and infection of the host under suitable predisposing conditions [1].

Candida albicans secretes hydrolytic enzymes, such as lipases, proteases, phospholipases, and hemolysin, which are considered to be integral to their virulence and pathogenesis. Expression of several genes, including ALS1, ALS3, ECE1, and $H W P 1$, is required for systemic candidiasis [2]. In particular, the gene hyphal wall protein 1 (HWP1) is known to encode a major $C$. albicans protein involved in several functions, including assemblage of cell wall, intracellular signaling, and hyphal expansion by cross-linking to the glucans of cell wall $[3,4]$. Moreover, $h w p 1$ promotes binding of Candida to epithelial cells, as the initial step of colonization and causes virulence in systemic candidiasis [4]. Genetics of C. albicans as a diploid fungal pathogen has been studied by many researchers $[5,6]$. C. albicans has two homozygous and heterozygous strains at the $h w p 1$ locus. One of the methods for the discrimination of these two strains is the amplification of this gene, whose homozygous strains produce one DNA fragment at 941 base pair (bp) while heterozygous strains produce two fragments in either 941 or $839 \mathrm{bp}$ [7-10]. One of the most common kinds of candidiasis in women is Candida vaginitis and Candida 
albicans which is reported as the most causative agent $[7,11]$. It occurs when Candida superficially penetrates into the mucosal layer of the vagina and causes an inflammatory response. The severity of signs and symptoms is typically different in patients [11], and secretion of enzymes as virulence factor is responsible for the severity of symptoms in patients suffering from Candida vaginitis [12].

According to Mucci et al. [8], in comparison with symptomatic and asymptomatic patients, there is no significant difference in the prevalence rate of homozygous and heterozygous $C$. albicans strain isolates.

Since the distribution of homozygous and heterozygous strains of $C$. albicans almost exists in the vaginal area, knowing the severity of enzyme secretion in these strains as a virulence factor might be effective in clinical manifestation of patients. The aim of this study was the comparative analysis of phospholipase, proteinase, and hemolysin activity among the homozygous and heterozygous strains of Candida vaginitis isolates.

\section{Materials and Methods}

2.1. Candida albicans Strain Isolates. In this study, a total of 60 stocks of Candida albicans species consisting of 30 homozygous and 30 heterozygous strains, which were previously recovered from Candida vaginitis patients (ethical code: IR.SUMS.REC.1397.380), were examined. These two strains were identified as homozygous and heterozygous strains level by molecular method previously [13] and kept in $-20^{\circ} \mathrm{C}$ condition. The isolates were subcultured on Sabouraud dextrose agar (Merck, Germany) before using.

2.2. Proteinase Secretion Activity. The proteinase secretion was detected by bovine serum albumin medium (BSA) according to Dagdeviren et al. [14]. Six-millimeter filter paper discs were dipped into a suspension of strains with a density of approximately $10^{7}$ yeast. $\mathrm{mL}^{-1}$ and applied to the BSA medium. The plates were incubated at $30^{\circ} \mathrm{C}$ for 6 days. After incubation, the diameter of opaque zones around the discs was considered as proteinase production. Precipitation zone $(\mathrm{Pz})$ was measured and expressed as negative $(-)$ for no clearance, positive $(+)$ for mild, double positive $(++)$ for moderate, triple positive $(+++)$ for strong, and four positives $(++++)$ for very strong enzyme activity. The standard strain of C. albicans (ATCC10261) was used as a positive control.

2.3. Phospholipase Secretion Activity. The plate method was used for the estimation of phospholipase activity according to Price et al. [15]. The medium was prepared by adding $5.5 \mathrm{~g}$ $\mathrm{CaCl}_{2}$ and $58.4 \mathrm{~g} \mathrm{NaCl}$ to Sabouraud dextrose agar (Merck, Germany). Then sterile egg yolk was centrifuged at $5,000 \times \mathrm{g}$ for $30 \mathrm{~min}$, followed by adding $20 \mathrm{~mL}$ of supernatant to the cooled medium. Two McFarland turbidity yeast suspension was prepared; $10 \mu \mathrm{L}$ yeast suspension was spot-inoculated on the plate medium and incubated at $37^{\circ} \mathrm{C}$ for up to 5 days. A precipitate zone around the colonies was measured, and precipitation zone was expressed as $\mathrm{Pz}$ value from 1 to 4 as follows: $\mathrm{Pz} 1$ (negative), $\mathrm{Pz} 1+(0.9-1), \mathrm{Pz} 2+(0.89-0.80), \mathrm{Pz}$
$3+(0.79-0.70)$, and $\mathrm{Pz} 4+(\geq 0.69)$. The standard strain of $C$. albicans (ATCC10261) was used as a positive control.

2.4. Hemolysin Activity. Blood plate assay was used for hemolytic activity as described by Yigit and Aktas [16]. Sheep blood Sabouraud dextrose agar was prepared by adding $7 \% \mathrm{v} / \mathrm{v}$ of fresh sheep blood and 3\% w/v of glucose. Next, 2 McFarland turbidity suspension was prepared. Then, 10 microliters of inoculation was spotted on sheep blood SDA plates and incubated at $37^{\circ} \mathrm{C}$ for 48 hours. A ring of lysis around the colonies was considered for hemolytic activity: complete (beta) in case of totally translucent ring, incomplete (alpha) in case of greenish-black halo, or no hemolysis (gamma or none). Candida albicans ATCC10261 was used as a positive control.

2.5. Statistical Analysis. Results were analyzed using the SPSS (Statistical Package for the Social Sciences) program. Fisher exact test $p$ value $<0.05$ was considered statistically significant.

\section{Results}

3.1. Results of Homozygous Strain Tests. Phospholipase activity was seen in $83.3 \%$ of the strains with $\mathrm{Pz}=0.59(0.38-1)$. Also, $66.7 \%$ of the strains were strong phospholipase producers.

All the strains were strong proteinase producers (100\%) with $\mathrm{Pz}=0.45(0.2-0.85)$ (Figure 1).

Hemolysin activity was seen in $100 \%$ of the strains considering the beta hemolysis pattern. More details are available in Table 1.

3.2. Results of Heterozygous Strain Tests. It was observed that $96 \%$ of the strains were phospholipase producers with $\mathrm{Pz}=0.63(0.3-1)$ and $76.7 \%$ were strong producers (Figure 1).

Proteinase activity was seen in $100 \%$ of the strains with $\mathrm{Pz}=0.46(0.3-0.6)$, and $90 \%$ of the strains were strong producers.

All of the strains had hemolysin activity (100\%), and beta hemolysis pattern was seen in $96.7 \%$ of the strains. More details are presented in Table 1.

3.3. Results of Statistical Analysis. Despite the variety of the test results in the two groups, statistical analysis using Fisher exact test reveals no correlation between the two strains in phospholipase activity $(p=0.29)$, proteinase activity $(p=0.23)$, and hemolysin activity $(p=1)$.

\section{Discussion}

Recently, fungal infections have represented a serious public health problem that needs urgent attention as they cause more than 1.5 million deaths worldwide each year. Candida albicans is one of the most common reasons of Candida infections that can cause both superficial and disseminated 

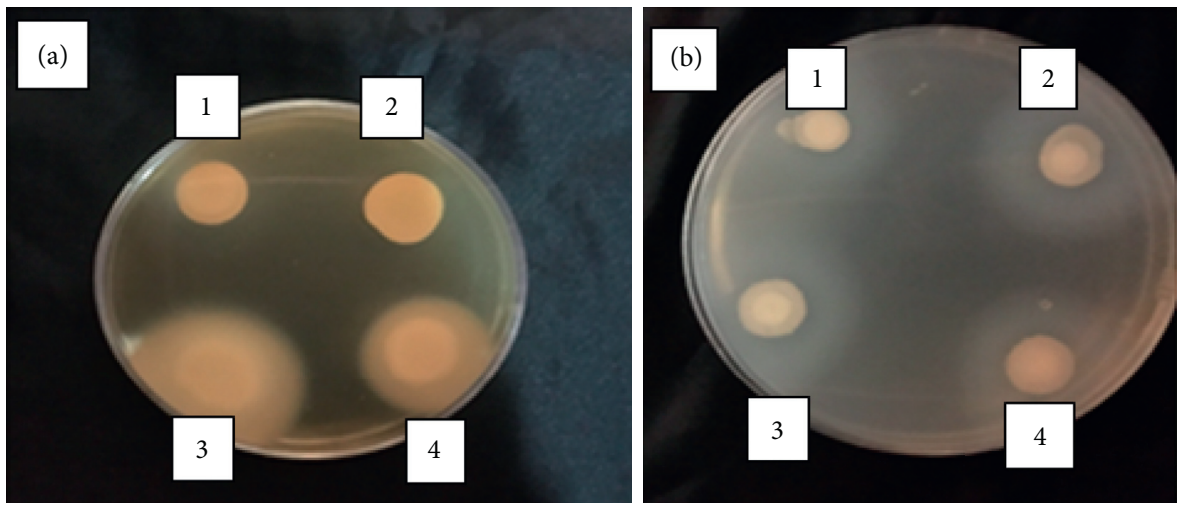

Figure 1: Phospholipase (a) and proteinase (b) activity. (a) Homozygous strain: spots 1, 3 (neg-pos); heterozygous strain: spots 2, 4 (negpos). (b) Homozygous and heterozygous strains: discs 1-4 (positive).

TABLE 1: Comparison of virulence factors in heterozygous and homozygous strains of vaginal Candida albicans species.

\begin{tabular}{|c|c|c|c|c|c|c|}
\hline \multirow{3}{*}{ Strains } & \multicolumn{5}{|c|}{ Virulence factors } & \multirow{3}{*}{ Total } \\
\hline & \multirow{2}{*}{ Phospholipase/Pz mean (range) } & \multirow{2}{*}{ Proteinase/Pz mean (range) } & \multicolumn{3}{|c|}{ Hemolysin (\%) } & \\
\hline & & & $\alpha$ & $\beta$ & $\gamma$ & \\
\hline Homozygous & $0.59(0.38-1)$ & $0.45(0.2-0.8)$ & - & 100 & - & 30 \\
\hline Heterozygous & $0.63(0.3-1)$ & $0.46(0.3-0.6)$ & - & 96.7 & 3.3 & 30 \\
\hline
\end{tabular}

infections. Candida dubliniensis and C. africana are other two yeast species that are closely related to C. albicans, which are named as C. albicans species complex [7, 17]. One of the best methods for discrimination of these species is the amplification of $h w p 1$ gene. By this method, three different sizes of DNA fragments that help identify these species are produced $[12,18]$. There are many studies about the role of hwpl gene in Candida pathogenesis and morphogenesis as an important risk factor. Tsuchimori et al. [19] investigated the role of this gene as a virulence factor and reported a reduction in virulence during exposing a homozygous hwp1 null mutant of C. albicans in infected mice. Moreover, homozygous and heterozygous strains of $C$. albicans could be discriminated from each other during amplification of $h w p 1$ locus by producing one or two DNA fragment sizes [8].

Candida vaginitis, as one of the most common Candida infections in women, is caused by many species of Candida, especially C. albicans. Candida vaginitis is strongly influenced by multiple factors such as hormone levels, type of organism, physiological condition, diabetes, antibiotic usage, and commensal bacteria. The secretion of extracellular hydrolytic enzymes, biofilm formation, phenotypic switching, adherence to host tissue, and many other factors have been listed as virulence factors and proposed to be involved in pathogenicity of Candida species [20, 21]. According to a study by Mucci et al. [8], both homozygous and heterozygous strains were isolated from women with vaginitis. If the secreted exoenzymes had been different between these two strains, this could probably have been responsible for the severity of symptoms in vaginal candidiasis patients. To our knowledge, there is no data about the evaluation of exoenzymes as virulence factors in these strains. The result of our data profile in phospholipase activity demonstrated that $66.7 \%$ of homozygous and $76.78 \%$ of heterozygous strains were high enzymatic activity producers, with no significant statistical difference between the two strains in the secretion of this enzyme.

Aspartyl proteinase could contribute to tissue damage during vaginal candidiasis infection while the other enzymes caused different tissue damage as well [22]. Regarding proteinase activity, all of the homozygous and heterozygous strains produced a high level of this exoenzyme, and these results indicate that proteinase in both strains can be considered as a virulence factor in the development of symptoms. Our results revealed the fact that both strains have the ability to secrete extracellular enzymes in a different range of severity.

Hemolysin activity was almost similar in the two strains, with heterozygous strain showing lower activity than the other strain. According to our study that showed no significant correlation between pathogenicity mechanisms of these strains and with this huge variability in causing of Candida vaginitis, it is necessary to consider other conditions of clinical symptoms in patients.

Reducing symptoms in vaginal candidiasis is spotted as treatment. There are several oral and topical antifungals and some other supplementary components such as phytol (acyclic diterpene alcohol) and avarol (marine natural product) used for this purpose [23, 24]. Centers of Disease Control and Prevention (CDC) recommended oral fluconazole as first-line therapy for vaginal candidiasis. On the other hand, topical imidazoles (i.e., econazole, clotrimazole, miconazole, and ketoconazole) are used and have noticeable effect [25]. Combination therapy of this conventional azole therapy and this supplementary agent may have a synergistic 
effect in treatment of vaginal candidiasis and could reduce symptoms [26].

Despite the statistical analysis results on exoenzyme activity, which showed no correlation between the two strains, we believe that in the case of increasing the number of samples, the statistical data could be statistically significant.

\section{Conclusion}

In this study, comparative analysis of virulence factors demonstrated that both of the strains could express exoenzymes as virulence factor in different ranges. There were no significant differences in virulence factors and pathogenesis of the two strains. Therefore, according to our data, we could not suggest any relation between the severity of symptoms in vaginal candidiasis patients and the type of these strains. However, more samples need to be examined to confirm our data.

\section{Data Availability}

The data used to support the findings of this study were supplied by the Deputy of Research and Technology of Shiraz University of Medical Sciences under license and so cannot be made freely available. Requests for access to these data should be sent to Keyvan Pakshir (pakshirk@ gmail.com).

\section{Disclosure}

This study was extracted from an M.S. thesis by Sahar Sheykhi.

\section{Conflicts of Interest}

The authors report no conflicts of interest.

\section{Acknowledgments}

This study was funded by the Vice-Chancellor for Research and Technology of Shiraz University of Medical Sciences, Shiraz, Iran (Grant no. 16724).

\section{References}

[1] M. V. Poornima and K. Vidyalakshmi, "Clinico-microbiological study of Candida infections focusing on risk factors, species identification, virulence factors and antifungal susceptibility patterns," National Journal of Laboratory Medicine, vol. 8, no. 3, pp. MO08-MO13, 2019.

[2] A. Islam, Characterizing Genes of Candida albicans Involved in Invasiveness, Mating and Genotoxic Stress Response, Concordia University, Montreal, Canada, 2018.

[3] A. P. V. De Melo, D. L. Zuza-Alves, W. P. Da Silva-Rocha et al., "Virulence factors of Candida spp. obtained from blood cultures of patients with candidemia attended at tertiary hospitals in Northeast Brazil," Journal de Mycologie Médicale, vol. 29, no. 2, pp. 132-139, 2019.

[4] J. F. Staab, S. D. Bradway, P. L. Fidel et al., "Adhesive and mammalian transglutaminase substrate properties of Candida albicans Hwp1,” Science, vol. 283, no. 5407, pp. 1535-1538, 1999.

[5] S. M. Noble and A. D. Johnson, "Genetics of Candida albicans, a diploid human fungal pathogen," Annual Review of Genetics, vol. 41, no. 1, pp. 193-211, 2007.

[6] J. Berman and P. E. Sudbery, "Candida albicans: a molecular revolution built on lessons from budding yeast," Nature Reviews Genetics, vol. 3, no. 12, pp. 918-931, 2002.

[7] Y. Shan, S. Fan, X. Liu, and J. Li, "Prevalence of Candida albicans-closely related yeasts, Candida africana and Candida dubliniensis, in vulvovaginal candidiasis," Medical Mycology, vol. 52, no. 6, pp. 636-640, 2014.

[8] M. J. Mucci, M. L. Cuestas, M. F. Landanburu, and M. T. Mujica, "Prevalence of Candida albicans, Candida dubliniensis and Candida africana in pregnant women suffering from vulvovaginal candidiasis in Argentina," Revista Iberoamericana de Micología, vol. 34, no. 2, pp. 72-76, 2017.

[9] J. R. Köhler and G. R. Fink, "Candida albicans strains heterozygous and homozygous for mutations in mitogen-activated protein kinase signaling components have defects in hyphal development," Proceedings of the National Academy of Sciences, vol. 93, no. 23, pp. 13223-13228, 1996.

[10] S.-H. Liang and R. J. Bennett, "The impact of gene dosage and heterozygosity on the diploid pathobiont Candida albicans," Journal of Fungi, vol. 6, no. 1, p. 10, 2019.

[11] J. D. Sobel, "Pathogenesis and treatment of recurrent vulvovaginal candidiasis," Clinical Infectious Diseases, vol. 14, no. Supplement_1, pp. S148-S153, 1992.

[12] K. Pakshir, M. Bordbar, K. Zomorodian et al., "Evaluation of CAMP-like effect, biofilm formation, and discrimination of Candida africana from vaginal Candida albicans species," Journal of Pathogens, vol. 2017, Article ID 7126258, 5 pages, 2017.

[13] K. Pakshir, S. Sheykhi, K. Zomorodian et al., "Evaluation of biofilm formation in the homozygous and heterozygous strains of vaginal Candida albicans isolates," Current Medical Mycology, vol. 5, no. 2, p. 37, 2019.

[14] M. Dagdeviren, N. Cerikcioglu, and M. Karavus, "Acid proteinase, phospholipase and adherence properties of Candida parapsilosis strains isolated from clinical specimens of hospitalised patients," Mycoses, vol. 48, no. 5, pp. 321-326, 2005.

[15] M. F. Price, I. D. Wilkinson, and L. O. Gentry, "Plate method for detection of phospholipase activity in Candida albicans," Medical Mycology, vol. 20, no. 1, pp. 7-14, 1982.

[16] N. Yigit and E. Aktas, "Comparison of the efficacy of different blood medium in determining the hemolytic activity of Candida species," Journal de Mycologie Médicale, vol. 19, no. 2, pp. 110-115, 2009.

[17] O. Romeo and G. Criseo, "Molecular epidemiology of Candida albicans and its closely related yeasts Candida dubliniensis and Candida africana," Journal of Clinical Microbiology, vol. 47, no. 1, pp. 212-214, 2009.

[18] R. Gumral, B. Sancak, A. B. Guzel, M. A. Saraçlı, and M. Ilkit, "Lack of Candida africana and Candida dubliniensis in vaginal Candida albicans isolates in Turkey using HWP1 gene polymorphisms," Mycopathologia, vol. 172, no. 1, pp. 73-76, 2011.

[19] N. Tsuchimori, L. L. Sharkey, W. A. Fonzi, S. W. French, J. E. Edwards, and S. G. Filler, "Reduced virulence of HWP1deficient mutants of Candida albicans and their interactions with host cells," Infection and Immunity, vol. 68, no. 4, pp. 1997-2002, 2000.

[20] S. Dabiri, M. Shams-Ghahfarokhi, and M. Razzaghi-Abyaneh, "Comparative analysis of proteinase, phospholipase, 
hydrophobicity and biofilm forming ability in Candida species isolated from clinical specimens," Journal de Mycologie Médicale, vol. 28, no. 3, pp. 437-442, 2018.

[21] M. Jafari, S. Salari, K. Pakshir, and K. Zomorodian, "Exoenzyme activity and possibility identification of Candida dubliniensis among Candida albicans species isolated from vaginal candidiasis," Microbial Pathogenesis, vol. 110, pp. 73-77, 2017.

[22] M. Schaller, M. Bein, H. C. Korting et al., "The secreted aspartyl proteinases Sap1 and Sap2 cause tissue damage in an in vitro model of vaginal candidiasis based on reconstituted human vaginal epithelium," Infection and Immunity, vol. 71, no. 6, pp. 3227-3234, 2003.

[23] B. Pejin, A. Savic, M. Sokovic et al., "Furtherin vitroevaluation of antiradical and antimicrobial activities of phytol," Natural Product Research, vol. 28, no. 6, pp. 372-376, 2014.

[24] B. Pejin, A. Ciric, D. Markovic, G. Tommonaro, and M. Sokovic, "In vitroavarol does affect the growth of Candida sp.” Natural Product Research, vol. 30, no. 17, pp. 1956-1960, 2016.

[25] H. L. Paladine and U. A. Desai, "Vaginitis: diagnosis and treatment," American Family Physician, vol. 97, no. 5, pp. 321-329, 2018.

[26] B. Pejin, C. Iodice, G. Tommonaro et al., "Further in vitro evaluation of antimicrobial activity of the marine sesquiterpene hydroquinone avarol," Current Pharmaceutical Biotechnology, vol. 15, no. 6, pp. 583-588, 2014. 